\title{
Effects of chronic Porphyromonas gingivalis lipopolysaccharide infusion on skeletal muscles in mice
}

\author{
Naoya Kawamura ${ }^{1,2} \cdot$ Yoshiki Ohnuki $^{1} \cdot$ Ichiro Matsuo $^{1,2} \cdot$ Kenji Suita $^{1} \cdot$ Misao Ishikawa $^{3} \cdot$ Yasumasa Mototani $^{1}$. \\ Kouichi Shiozawa ${ }^{1}$. Aiko Ito $^{1,4} \cdot$ Yuka Yagisawa $^{1,4} \cdot$ Yoshio Hayakawa $^{1,5} \cdot$ Megumi Nariyama $^{6} \cdot$ Daisuke Umeki $^{4}$. \\ Yuko Ujiie $^{2} \cdot$ Kazuhiro Gomi ${ }^{2} \cdot$ Satoshi Okumura' ${ }^{1}$
}

Received: 30 December 2018 / Accepted: 23 February 2019 / Published online: 8 March 2019

(c) The Physiological Society of Japan and Springer Japan KK, part of Springer Nature 2019

\begin{abstract}
Periodontitis, which is caused by various oral organisms, predominantly affects adults, and is one of the main causes of tooth loss, as well as leading to progression of numerous systemic diseases. However, its relationship to sarcopenia (agingassociated degenerative loss of skeletal muscle mass and function) remains unclear. The aim of this study was to investigate the effects of Porphyromonas gingivalis lipopolysaccharide (PG-LPS) on skeletal muscle in mice, and to establish the underlying mechanisms. Mice (C57BL/6) were injected with PG-LPS $(0.8 \mathrm{mg} / \mathrm{kg} /$ day) for 4 weeks. This treatment significantly decreased the weight of fast-twitch skeletal muscles (masseter and tibialis anterior muscles), but not that of slow-twitch skeletal muscle (soleus muscle). The area of fibrosis was significantly increased in masseter muscle, but remained unchanged in the other two muscles. The number of apoptotic myocytes was significantly increased (approximately eightfold) in masseter muscle. These data suggest that persistent subclinical exposure to PG-LPS might reduce the size of fast-twitch skeletal muscle, but not slow-twitch skeletal muscle. Masseter muscle appears to be especially susceptible to the adverse effects of PG-LPS, because muscle remodeling (muscle fibrosis and myocyte apoptosis) was induced solely in masseter muscle. Thus, periodontitis might be one of the major causes of oral sarcopenia.
\end{abstract}

Keywords Lipopolysaccharide $\cdot$ Periodontitis $\cdot$ Sarcopenia $\cdot$ Apoptosis $\cdot$ Fibrosis $\cdot$ Signal transduction

\section{Introduction}

Satoshi Okumura

okumura-s@tsurumi-u.ac.jp

1 Department of Physiology, Tsurumi University School of Dental Medicine, 2-1-3 Tsurumi, Tsurumi-ku, Yokohama 230-8501, Japan

2 Department of Periodontology, Tsurumi University School of Dental Medicine, Yokohama 230-8501, Japan

3 Department of Oral Anatomy, Tsurumi University School of Dental Medicine, Yokohama 230-8501, Japan

4 Department of Orthodontics, Tsurumi University School of Dental Medicine, Yokohama 230-8501, Japan

5 Department of Dental Anesthesiology, Tsurumi University School of Dental Medicine, Yokohama 230-8501, Japan

6 Department of Pediatric Dentistry, Tsurumi University School of Dental Medicine, Yokohama 236-8501, Japan
Inflammation that extends deep into the tissues and causes loss of supporting connective tissue and alveolar bone is known as periodontitis. Periodontitis results in the formation of soft tissue pockets or deepened crevices between the gingiva and tooth root. Release of components such as lipopolysaccharides by the causative organisms causes a heightened host inflammatory response and is thought to contribute to the body's overall inflammatory burden, worsening conditions such as diabetes mellitus, cardiovascular disease, stroke, pulmonary disease and adverse pregnancy outcome [1]. According to a recent report by Centers for Disease Control and Prevention (CDC) about one in two American adults aged 30 or more (64.7 million people) have mild, moderate or severe periodontitis. Moreover, in adults aged 65 and older, the prevalence is as high as $70 \%$, suggesting that periodontitis is ubiquitous in elderly individuals [2].

Age-related inflammation is associated with reductions in skeletal muscle size and function (sarcopenia), leading 
to a loss of independence and reduced quality of life [3]. In the context of skeletal muscle inflammation, inflammatory cytokines [tumor necrosis factor (TNF)- $\alpha$ and interleukin- 6 (IL-6)] are strongly induced by circulating LPS [4] and indeed, they are generally elevated in aged individuals [5, 6]. Increased serum levels of TNF- $\alpha$ and IL-6 are involved in the pathogenesis of periodontitis [7]. Several studies have indicated that improving oral care through mechanical or chemical control of dental plaque biofilm formation might contribute to the prevention of systemic inflammation and cardiovascular disease [8]. These data suggest that periodontitis might lead to reduced skeletal muscle size and function, i.e., sarcopenia.

Porphyromonas gingivalis lipopolysaccharide (PG-LPS), a major pathogenic factor for periodontitis, was recently demonstrated to induce cognitive impairment in mice via the activation of Toll-like 4 receptor (TLR4) and the increase of inflammatory cytokines (TNF- $\alpha$, IL- $1 \beta$, IL-6, and IL-8) in the cortex [9]. However, to our knowledge, there has been no report on the effects of PG-LPS on skeletal muscle in vivo. We hypothesized that PG-LPS might play an important role in the development of sarcopenia. In this study, we tested this hypothesis in mice treated with PG-LPS at a dose equivalent to the circulating level in periodontitis patients [10].

\section{Materials and methods}

\section{Mice and experimental protocols}

All experiments were performed on male 12-week-old C57BL/6 mice obtained from CLEA Japan (Tokyo, Japan). Mice were group-housed at $23{ }^{\circ} \mathrm{C}$ under a $12-12$ light/dark cycle with lights on at 8:00 a.m. Both food and water were available ad libitum. This study was approved by the Animal Care and Use Committees of Tsurumi University.

PG-LPS (Wako, Osaka, Japan) was dissolved in saline to prepare a $0.6 \mathrm{mg} / \mathrm{ml}$ stock solution, and the appropriate volume of this solution to provide the desired dose $(0.8 \mathrm{mg} /$ $\mathrm{kg}$ ) was added to $0.2 \mathrm{ml}$ of saline to prepare the solution for intraperitoneal (i.p.) injection once daily for 4 weeks; control mice received an identical volume of saline only (Fig. 1a)
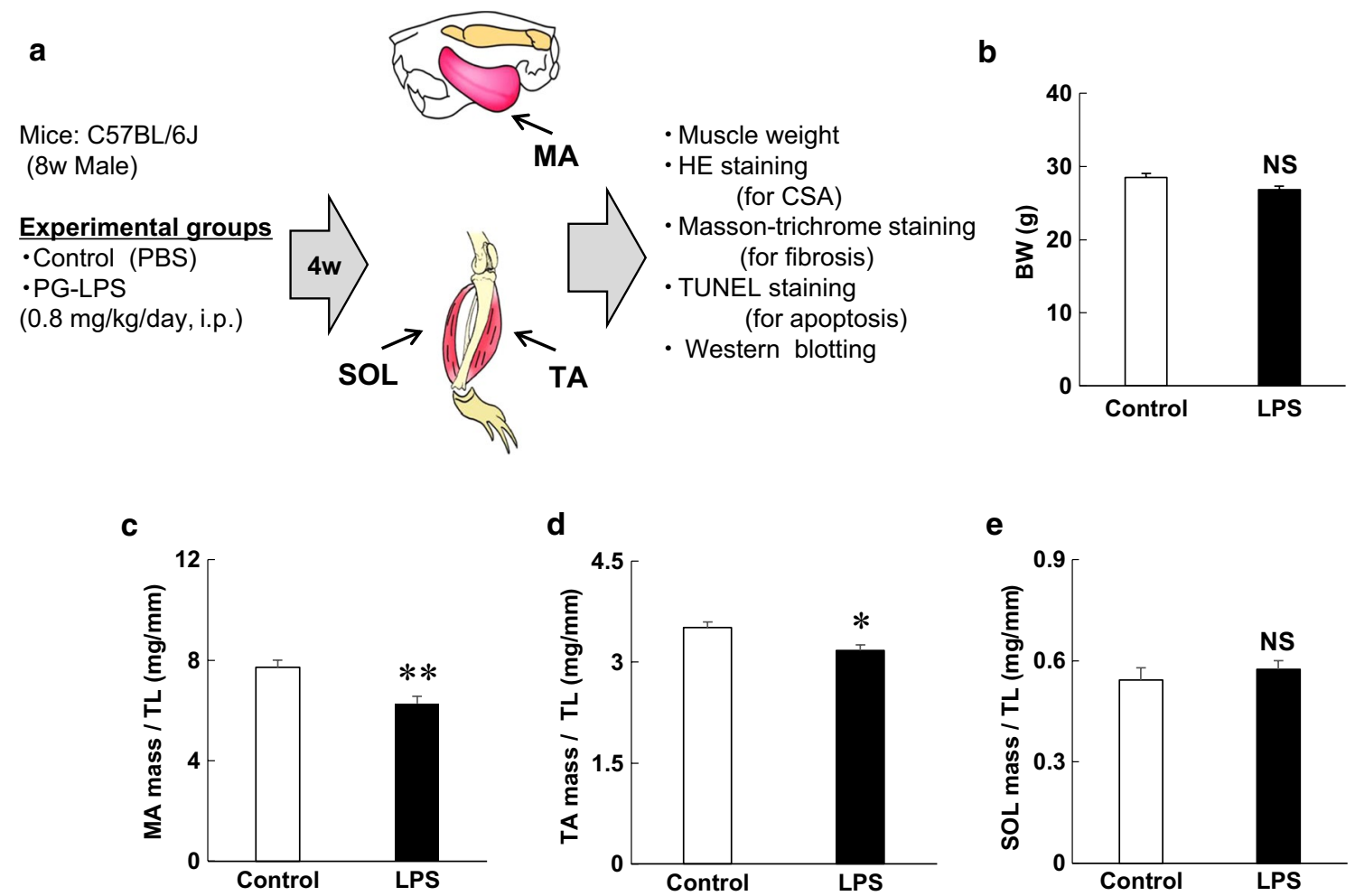

Fig. 1 Experimental procedure and changes of body and muscle weights during chronic PG-LPS infusion in mice. a PG-LPS was administered once daily for 4 weeks $(4 \mathrm{w})$ via intraperitoneal injection (i.p.) at a dose of $0.8 \mathrm{mg} / \mathrm{kg}$ in saline. Age-matched control mice (Control) received an identical volume of saline only. PG-LPS; Porphylomonas gingivalis lipopolysaccharide, MA; masseter muscle, TA; tibials anterior muscle, SOL; soleus muscle, CSA; cross- sectional area, b Control and PG-LPS groups showed similar body weight (BW) at 4 weeks after the PG-LPS infusion $(P=\mathrm{NS}$, not significant). c-e Masseter muscle (MA) weight per tibia length (TL) ratio (c) and tibialis anterior muscle weight (TA) per TL ratio (d) were significantly decreased by PG-LPS treatment. Soleus muscle (SOL) weight per TL ratio (e) was similar before and after PG-LPS treatment $\left({ }^{*} P<0.05,{ }^{* *} P<0.01\right.$ vs control) 
[10]. The dose of PG-LPS used in this study is consistent with the circulating levels in periodontitis patients, indicating that this model is not a sepsis model, and no mortality was observed [10]. After the completion of treatment, mice were anesthetized with isoflurane and the masseter (MA), tibialis anterior (TA) and soleus muscles (SOL) were excised and weighed, frozen in liquid nitrogen, and stored at $-80{ }^{\circ} \mathrm{C}$. The ratio of muscle mass $(\mathrm{mg})$ to tibia length (mm) was used as an index of muscle growth. After tissue extraction, the mice were killed by cervical dislocation [11].

\section{Cross-sectional area of muscle fibers}

The MA, TA and SOL, each excised as a whole muscle, were embedded in Tissue-Tek OCT compound (Sakura Finetec, Torrance, CA, USA) in a slightly stretched state so as to maintain a length close to the resting length (L0), and stored at $-80{ }^{\circ} \mathrm{C}$ until sectioning, as reported [12]. Cross sections $(10 \mu \mathrm{m})$ were cut from the middle portion of each muscle with a cryostat (CM1900, Leica Microsystems, Nussloch, Germany) at $-20{ }^{\circ} \mathrm{C}$. The sections were air-dried and fixed with $4 \%$ paraformaldehyde in $0.1 \mathrm{M}$ PBS (pH 7.5). The sections were then stained with hematoxylin and eosin (HE) and observed under a light microscope (BX61, Olympus Co., Tokyo, Japan). Micrographs were taken with a digital camera (DP-72, Olympus Co.) connected to a personal computer. The cross-sectional size of muscle fibers was evaluated by measuring the cross-sectional area (CSA) $[13,14]$. The CSAs of 100 muscle fibers in the superficial portion were measured with image analysis software (Image J 1.45) and averaged to obtain the mean values in each mouse.

\section{Evaluation of fibrosis}

Interstitial fibrosis was evaluated by Masson-trichrome staining using the Accustatin Trichrome Stain Kit (Sigma, St. Louis, Mo, USA) in accordance with the manufacturer's protocol. Interstitial fibrotic regions were quantified using image software analysis (Image $\mathrm{J} 1.45$ ) to determine the percentage of blue area in the Masson-trichrome-stained sections [15].

\section{Evaluation of apoptosis}

Apoptosis was determined by terminal deoxyribonucleotidyl transferase-mediated biotin-16-deoxyuridine triphosphate nick-end labeling (TUNEL) staining using an Apoptosis in situ Detection Kit (\#293-71501; Wako, Osaka, Japan). TUNEL-positive nuclei per field of view were manually counted in six sections of two groups (Control and PG-LPS) over a microscopic field of $20 \times$, averaged and expressed as the ratio of TUNEL-positive nuclei $(\%)[14,15]$. Limiting the counting of total nuclei and TUNEL-positive nuclei to areas with a true cross section of myocytes made it possible to selectively count only those nuclei that were clearly located within myocytes.

\section{Western blotting}

The MA excised from the mice (Fig. 1a) was homogenized in a Polytron (Kinematica AG, Lucerne, Switzerland) in icecold RIPA buffer (Thermo Fisher Scientific, Waltham, MA: 25 mM Tris- $\mathrm{HCl}$ (pH 7.6), $150 \mathrm{mM} \mathrm{NaCl}, 1 \% \mathrm{NP}-40,1 \%$ sodium deoxycholate, $0.1 \% \mathrm{SDS}$ ) without addition of inhibitors [16], and the homogenate was centrifuged at $13,000 \times g$ for $10 \mathrm{~min}$ at $4{ }^{\circ} \mathrm{C}$. The supernatant was collected and the protein concentration was measured using a DC protein assay kit (Bio-Rad, Hercules, CA). Equal amounts of protein $(5 \mu \mathrm{g})$ were subjected to $12.5 \%$ SDS-polyacrylamide gel electrophoresis and blotted onto $0.2 \mathrm{~mm}$ PVDF membrane (Millipore, Billerica, MA).

The primary antibodies against Akt (\#9272), phosphoAkt (Ser-473, \#9721), p44/42MAPK (ERK) (\#4695), phospho-ERK (Thr-202/Tyr-204, \#4370), microtubule-associated protein light chain 3 (LC3)-II (\#12741), p70-kDa ribosomal S6 kinase (p70S6 K) (\#9202), phospho-p70S6 K (Thr-389, \#9205), eukaryotic initiation factor 4E-binding protein 1 (4E-BP1) (\#9644), phospho-4E-BP1 (Thr-37/46, \#2855), Smad2/3 (\#8685) and phospho-Smad2 (Ser-465/467)/3 (Ser-423/425) (\#8828) were purchased from Cell Signaling Technology (Boston, MA, USA), while the primary antibodies against GAPDH (sc-25778) were purchased from Santa Cruz Biotechnology (Santa Cruz, CA, USA).

\section{Statistical analysis}

All data are expressed as the mean \pm SEM. Comparison of data was performed using Student's $t$-test. Differences were considered significant when $P<0.05$.

\section{Results}

\section{Effects of PG-LPS on body weight and weight of masseter, tibialis anterior and soleus muscles}

The control and PG-LPS groups showed similar body weight (BW) at 4 weeks after the PG-LPS infusion [Control $(n=6)$ vs PG-LPS $(n=6): 28 \pm 0.8 \mathrm{~g}$ vs $27 \pm 0.7 \mathrm{~g}, P=$ not significant (NS) vs control] (Fig. 1b). We next evaluated hypertrophy of MA (fast-twitch muscle) (Fig. 1c), TA (fasttwitch muscle) (Fig. 1d) and SOL (slow-twitch muscle) (Fig. 1e) in terms of the ratio of muscle weight per tibia length $(\mathrm{mg} / \mathrm{mm})$. This ratio was significantly decreased in fast-twitch muscle, i.e., MA [control $(n=5)$ vs PG-LPS $(n=5): 7.7 \pm 0.3 \mathrm{mg} / \mathrm{mm}$ vs $6.3 \pm 0.3 \mathrm{mg} / \mathrm{mm} P<0.01$ vs 
control] (Fig. 1c) and TA [control $(n=5)$ vs PG-LPS $(n=5)$ : $3.5 \pm 0.1 \mathrm{mg} / \mathrm{mm}$ vs $3.2 \pm 0.1 \mathrm{mg} / \mathrm{mm}, P<0.05$ vs control] (Fig. 1d), but not in SOL [control $(n=6)$ vs PG-LPS $(n=5)$ : $0.5 \pm 0.04 \mathrm{mg} / \mathrm{mm}$ vs $0.6 \pm 0.03 \mathrm{mg} / \mathrm{mm}, P=\mathrm{NS}$ ] (Fig. 1e).

These data suggest that PG-LPS treatment under the experimental conditions used in this study does not affect growth, but might induce muscle atrophy in fast-twitch muscle (MA and TA), though not in slow-twitch muscle (SOL).

\section{Effects of PG-LPS on CSA of MA, TA and SOL muscles}

Next, to confirm the difference between the effects of PGLPS on MA, TA and SOL muscle mass, we performed HE staining and measured the fiber CSA. The CSA was significantly decreased in the MA [control $(n=5)$ vs PGLPS $(n=5): 1664 \pm 61 \mu \mathrm{m}^{2}$ vs $1470 \pm 59 \mu \mathrm{m}^{2}, P<0.05$ vs control] (Fig. 2a) and TA muscles [control $(n=4)$ vs PGLPS $(n=4): 1711 \pm 42 \mu \mathrm{m}^{2}$ vs $1536 \pm 47 \mu \mathrm{m}^{2}, P<0.05$ vs control] (Fig. 2b). However, it was unchanged in SOL muscle [control $(n=6)$ vs PG-LPS $(n=6): 1332 \pm 50 \mu \mathrm{m}^{2}$ vs $1417 \pm 39 \mu \mathrm{m}^{2}, P=\mathrm{NS}$ vs control] (Fig. $2 \mathrm{c}$ ).

These data support the idea that PG-LPS treatment under the experimental conditions used in this study might induce atrophy in fast-twitch muscles (MA and TA), but not in slow-twitch muscle (SOL) (Fig. 1).

\section{Effects of PG-LPS on fibrosis in MA, TA and SOL muscles}

We next focused on skeletal muscle remodeling, which we evaluated in terms of fibrosis and myocyte apoptosis, because such remodeling might lead to progressive sarcopenia $[17,18]$. We examined the effects of PG-LPS on fibrosis in MA, TA and SOL muscles by means of Massontrichrome staining (Fig. 3). PG-LPS significantly increased the area of fibrosis in MA [control $(n=6)$ vs PG-LPS $(n=6): 2.5 \pm 0.2 \mu \mathrm{m}^{2}$ vs $4.3 \pm 0.3 \mu \mathrm{m}^{2}, P<0.01$ vs control] (Fig. 3a). However, PG-LPS did not alter the area of fibrosis in TA or SOL muscle [TA: control $(n=6)$ vs PG-LPS a

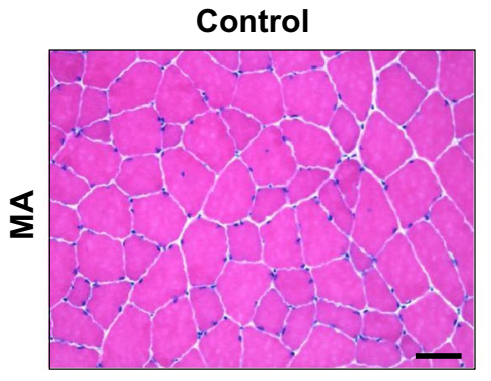

b

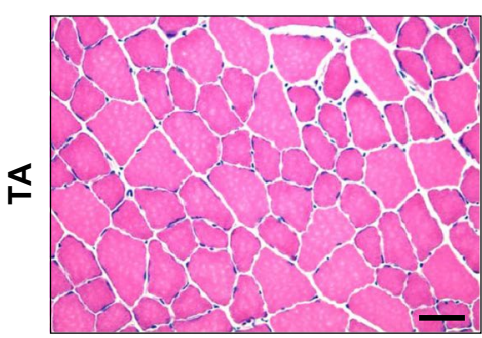

C

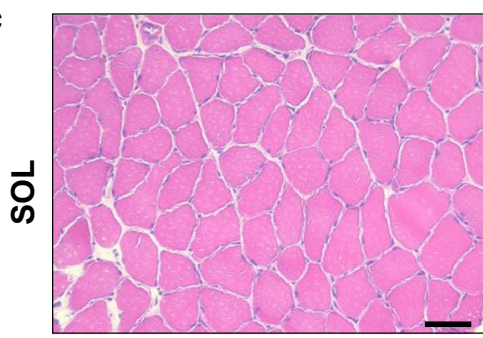

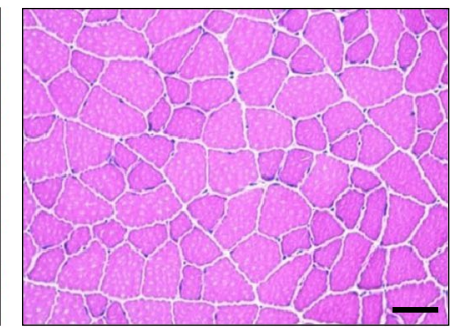
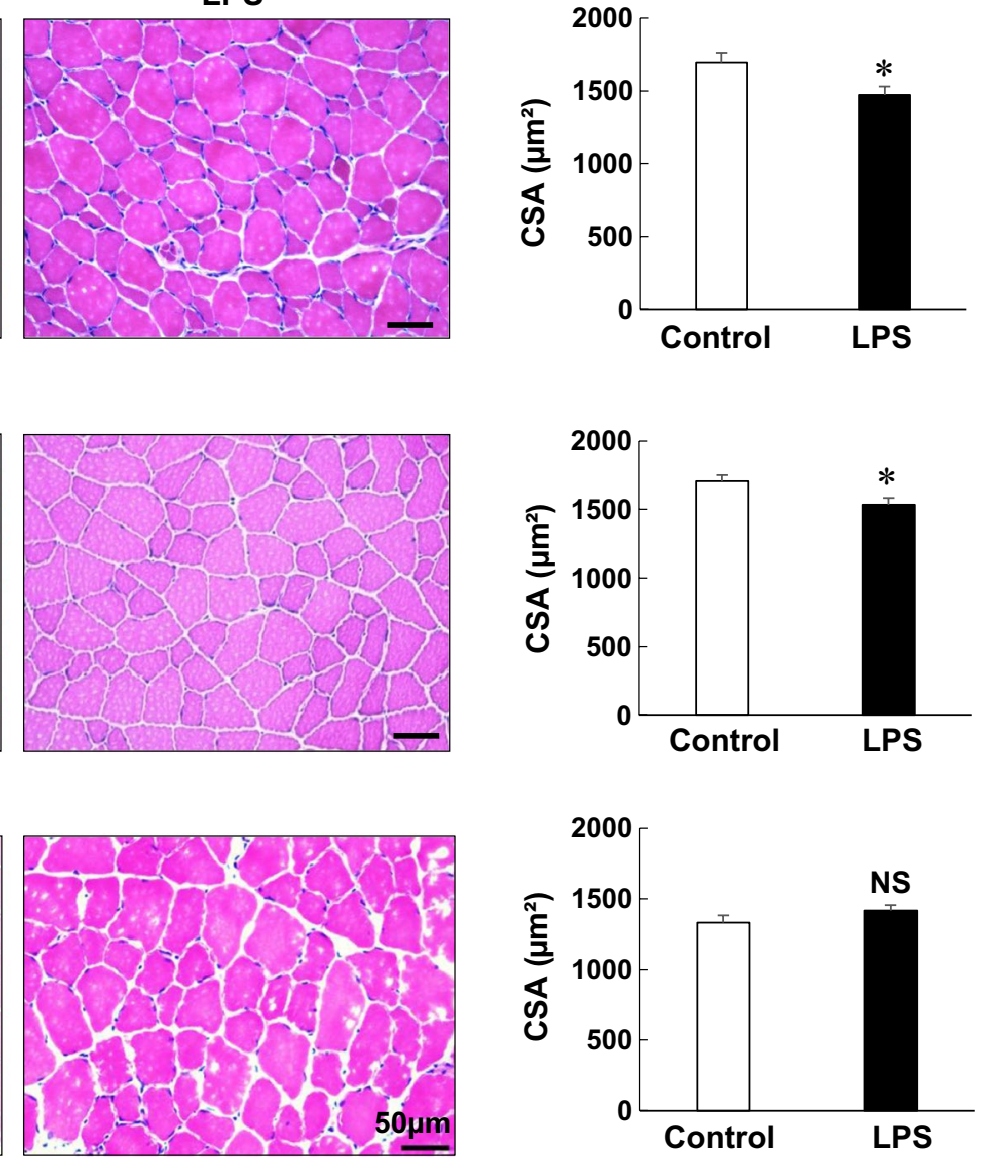

Fig. 2 Effects of PG-LPS on muscle weight. a-c Typical HE-stained sections of MA (a), TA (b) and SOL (c) muscles in the control (left) and PG-LPS groups (middle), in addition to cross-sectional area
(CSA) (right). CSA was significantly decreased by PG-LPS treatment in MA and TA muscles ( ${ }^{*} P<0.05$ vs control), but it remained unchanged in SOL muscle. Scale bars: $50 \mu \mathrm{m}$ 
a

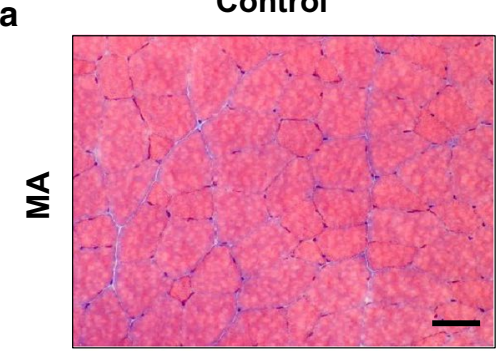

b

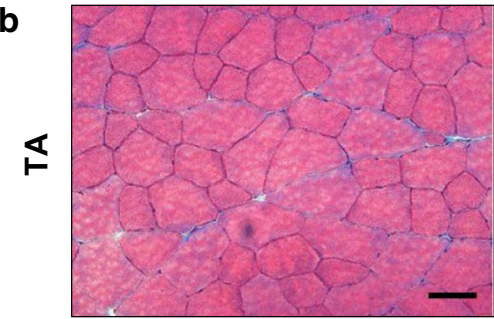

C

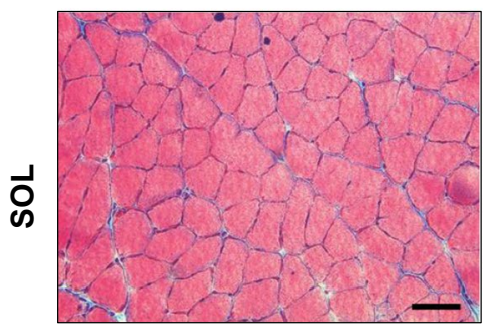

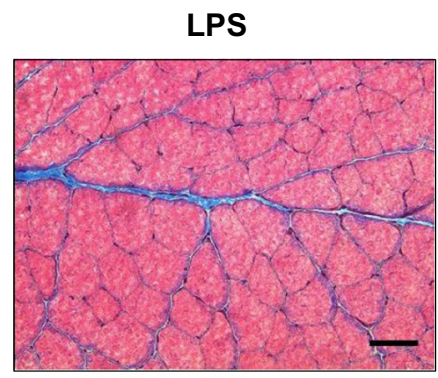
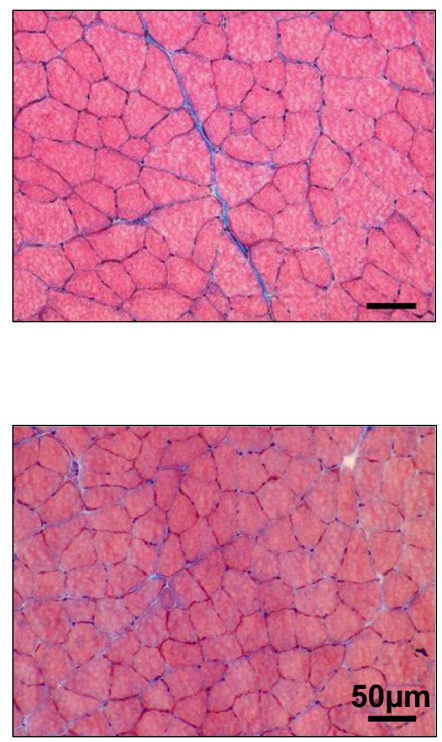
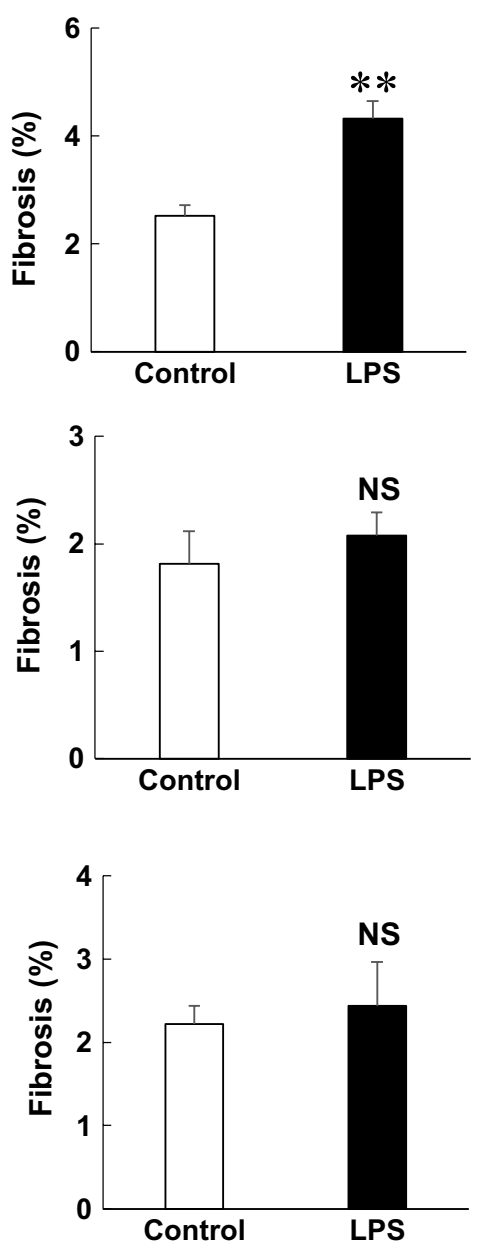

Fig. 3 Effects of PG-LPS on muscle fibrosis. a-c Representative images of Masson-trichrome-stained sections of MA (a), TA (b) and SOL (c) muscles in the Control (left) and PG-LPS groups (middle), in addition to the area of fibrosis (right). In the PG-LPS group, the

$(n=6): 1.8 \pm 0.3 \mu \mathrm{m}^{2}$ vs $2.1 \pm 0.2 \mu \mathrm{m}^{2}, P=\mathrm{NS}$ vs control; SOL: control $(n=6)$ vs PG-LPS $(n=6): 2.2 \pm 0.2 \mu \mathrm{m}^{2}$ vs $2.4 \pm 0.5 \mu \mathrm{m}^{2}, P=\mathrm{NS}$ vs control].

These data indicated that MA might be more susceptible to PG-LPS-mediated fibrosis, compared to lower limb muscles such as TA and SOL.

\section{Effects of PG-LPS on myocyte apoptosis in MA}

We next examined the effects of PG-LPS on myocyte apoptosis in MA by means of TUNEL staining (Fig. 4a). Myocyte apoptosis in MA was significantly increased by PG-LPS [control $(n=6)$ vs PG-LPS $(n=6): 0.9 \pm 0.06 \%$ vs $7.3 \pm 0.8 \%, P<0.01$ vs control] (Fig. $4 \mathrm{a}$ ).

These data indicated that PG-LPS might induce myocyte apoptosis in MA. area of fibrosis was significantly increased in the MA $\left(^{* *} P<0.01\right.$ vs control), but it remained unchanged in the TA and SOL. Scale bars: $50 \mu \mathrm{m}$

\section{ERK signaling was activated in the MA of PG-LPS-treated mice}

ERK activation was reported to be involved in LPS-mediated cardiac and hepatic failure via induction of fibrosis and apoptosis $[19,20]$. Therefore, we examined the amount of phospho-ERK (Thr-202/Tyr-204) in MA of PG-LPS-treated mice and found that ERK phosphorylation was significantly increased [control $(n=4)$ vs PGLPS ( $n=4): 100 \pm 11 \%$ vs $257 \pm 57 \%, P<0.05$ vs control] (Fig. 4b).

Thus, PG-LPS-mediated MA fibrosis and myocyte apoptosis might be mediated, at least in part, through the activation of TLR4-ERK signaling. 
a

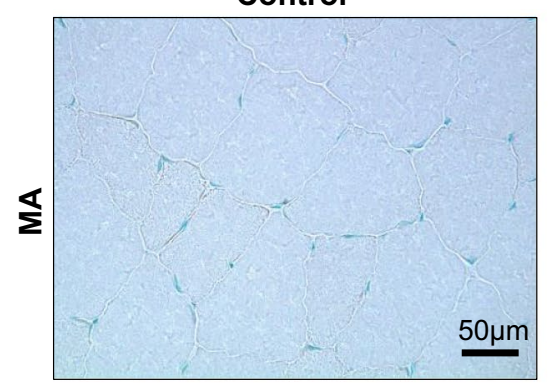

LPS

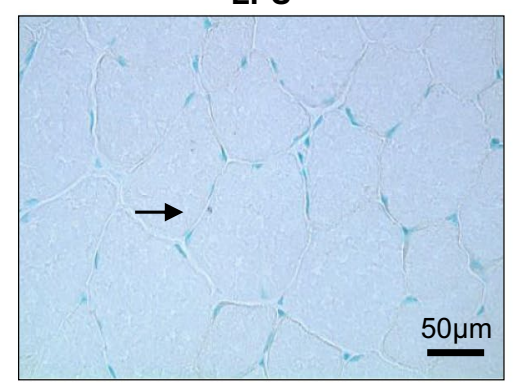

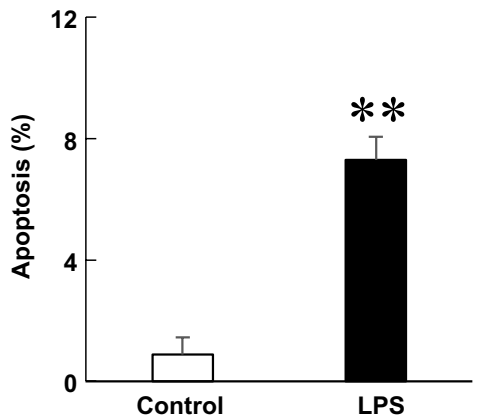

b
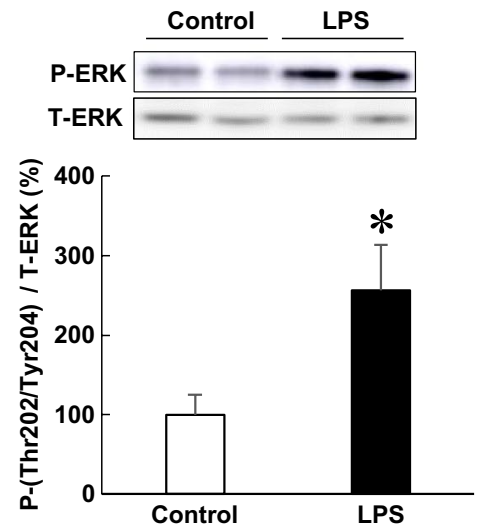

e

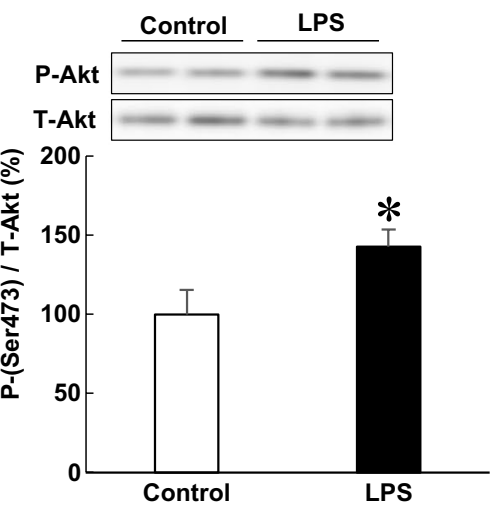

C
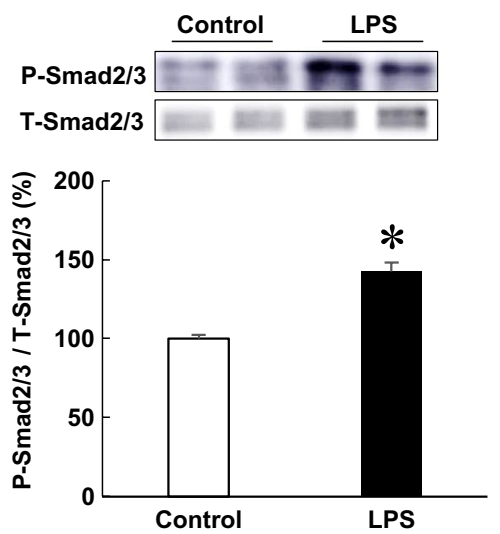

f

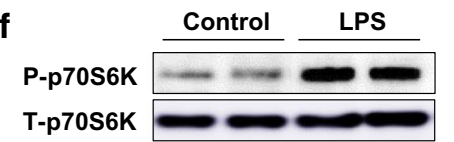

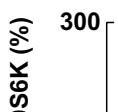

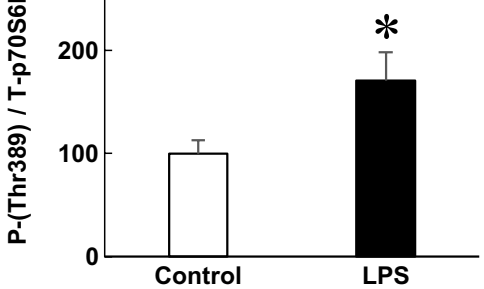

d
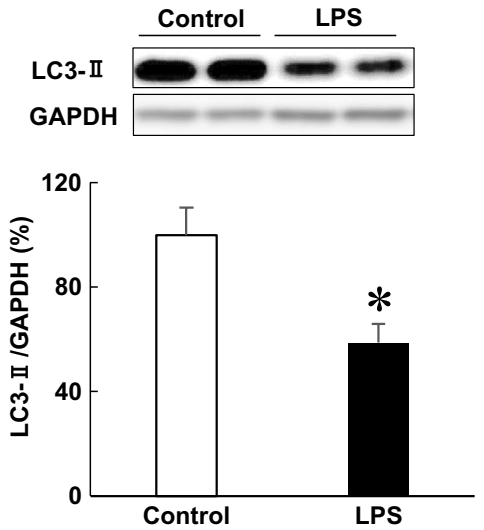

g
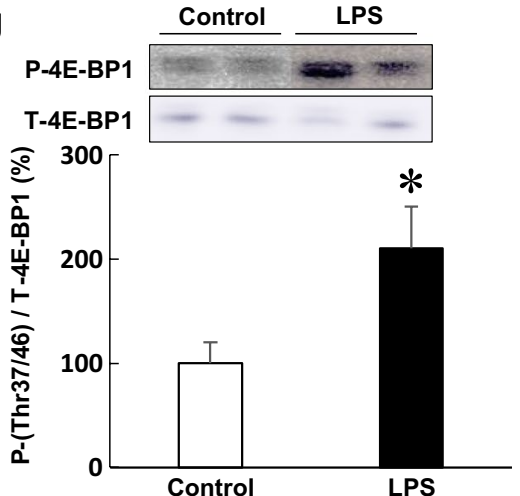

Fig. 4 Effects of PG-LPS on myocyte apoptosis and molecules involved in muscle remodeling and autophagy. a Representative images of TUNEL-stained sections of MA in the Control (left) and PG-LPS groups (right). Scale bars: $50 \mu \mathrm{m}$. Black arrow: TUNELpositive myocyte. The number of TUNEL-positive myocytes was significantly increased in the PG-LPS group (far right) $\left({ }^{* *} P<0.01\right.$ vs Control). b-g Expression of molecules involved in muscle remod-

\section{Smad2/3 phosphorylation was increased in the MA of PG-LPS-treated mice}

Smad $2 / 3$ phosphorylation is located downstream of TGF- $\beta$ signaling and is critical for the induction and maintenance of fibroblast activation in cardiac and skeletal muscle $[21,22]$. There is extensive evidence that activation of cells through LPS is mediated by TLR4 and other molecules including TGF- $\beta$-Smad2/3 [23, 24]. Therefore, we eling and autophagy: ERK (Thr-202/Tyr-204) (b), Smad2 (Ser463/467)/Smad (Ser-423/425) (c), LC3-II (d), Akt (Ser-473) (e), p70S6 K (Thr-389) (f) and 4E-BP1 (Thr-37/46) (g). Expression in the MA of the control group was taken as $100 \%$ in each case. Representative immunoblotting results are shown $\left({ }^{*} P<0.05,{ }^{* *} P<0.01\right.$ vs control). $P$ phosphorylated, $T$ total

examined the amount of phospho-Smad2 (Ser-463/467)/ Smad3 (Ser-423/425) in MA of PG-treated mice. We found that Smad2/3 phosphorylation was significantly increased in the MA of PG-LPS-treated mice [control $(n=6)$ vs PGLPS $(n=6): 100 \pm 2.6 \%$ vs $143 \pm 6.7 \%, P<0.01$ vs control] (Fig. 4c).

These data suggest that PG-LPS-induced MA fibrosis might be mediated through the activation of TGF- $\beta$-Smad $2 / 3$ signaling in addition to TLR4-ERK signaling. 


\section{Autophagy in MA was decreased by chronic PG-LPS infusion}

We next investigated the effects of PG-LPS on autophagy in the MA, because autophagy is important in maintaining muscle homeostasis physiologically and in response to stress [25]. We found that LC3-II, an autophagosome marker, was significantly decreased in the MA of PG-LPS-treated mice [control $(n=4)$ vs PG-LPS $(n=4): 100 \pm 10.5 \%$ vs $59 \pm 7.2 \%, P<0.05$ vs control] (Fig. 4 d).

These data suggest activation of autophagy in MA might be attenuated by PG-LPS [26].

\section{Akt/mTOR signaling was activated in MA of PG-LPS-treated mice}

We then examined the phosphorylation of Akt in MA, since the Akt-mTOR pathway directly phosphorylates 4E-BP1 at threonine $37 / 46$, as well as p70S6 $\mathrm{K}$ at threonine 389 , and these changes inhibit autophagy in cells, including skeletal myocytes [27].

We first examined the Akt phosphorylation (Ser-473) in MA and found that it was significantly increased in the PGLPS group [control $(n=6)$ vs PG-LPS $(n=6): 100 \pm 15.5 \%$ vs $143 \pm 11.0 \%, P<0.05$ vs control] (Fig. $4 \mathrm{e}$ ). We next examined the amounts phospho-p70S6 K (Thr-389) (Fig. 4f) and phospho-4E-BP1 (Thr-37/46) (Fig. 4g) in the MA of PG-LPS-treated mice, and found that both phosphorylations were significantly increased (p70S6 K (Thr-389): control $(n=6)$ vs PG-LPS $(n=6): 100 \pm 13.2$ vs $170 \pm 27.8 \%$, $P<0.05$; 4E-BP1 (Thr-37/46): control $(n=5)$ vs PG-LPS $(n=5): 100 \pm 20.4 \%$ vs $210 \pm 40.4 \%, P<0.05)$.

These data indicated that activation of Akt/mTOR/ p70S6 K or 4E-BP1 signaling might be important for MA remodeling through inhibition of autophagy during chronic PG-LPS exposure.

\section{Discussion}

In this study, we examined the effects of chronic PG-LPS infusion on fast-twitch (MA and TA) and slow-twitch (SOL) skeletal muscle in mice at a dose consistent with the circulating levels in periodontitis patients [10]. A significant reduction of muscle mass was observed in fast-twitch skeletal muscle (MA and TA), but not in slow-twitch muscle (SOL). Moreover, significant histological abnormalities, i.e., fibrosis and myocyte apoptosis, were also observed in MA, concomitantly with the decrease of muscle mass.

Sarcopenic dysphagia is a recently defined condition that is characterized by loss of mass and function of the swallowing muscles, including MA, and is also associated with loss of skeletal muscle mass and function throughout the body
[28]. Although it is not yet fully established whether sarcopenia is associated with periodontitis, another age-related disease, it was recently reported that reduced MA function was correlated with the severity of periodontitis [29]. Our findings here indicate that susceptibility to PG-LPS-induced muscle atrophy is dependent upon the muscle fiber-type, and fast-twitch muscle is especially susceptible, as is also the case for other muscle-wasting diseases, such as cancer cachexia, starvation, sepsis and acute diabetes [30].

Skeletal muscle consists of myofibers (the contractile part of the muscle), connective tissue or extracellular matrix (ECM), and the blood capillaries and nerves supplying the muscle [31]. ECM forms up to $10 \%$ of the skeletal muscle weight [32] and it plays a principal role in force transmission, maintenance, and repair of muscle fibers following injury [33]. Skeletal muscle has a high regeneration capability, but this can be compromised by excessive deposition of ECM, which results in muscle fibrosis [33]. The excessive deposition of fibrous tissue impairs muscle function, and affects muscle fiber regeneration after injury, as well as muscle susceptibility to re-injury [18]. Here, we found that muscle fibrosis, measured in terms of Masson-trichrome staining, was significantly increased in MA, but not in TA or SOL. This result indicates that the functional decline of MA might be more severe than that of lower limb muscles in mice treated with PG-LPS.

Autophagy is critical for maintaining skeletal muscle mass in both young and aged mice, and insufficient autophagy can lead to an accumulation of dysfunctional mitochondria, which promotes skeletal muscle remodeling and impairs muscle function [34]. Here, we found that expression of LC3-II protein, an autophagosome marker, was significantly decreased, while the Akt-mTOR pathway, a negative regulator of autophagy, was significantly activated in the MA of PG-LPS-treated mice [27].

This study, to our knowledge, is the first to demonstrate a relationship between a periodontics-associated pathogen and sarcopenia, in addition to uncovering the underlying mechanisms. Notably, we found that MA, a major swallowing-related muscle, appears to be more susceptible than TA or SOL muscles to the adverse effects of PG-LPS. This is important, because it has been reported that the consequences of dysphagia in hospitalized patients with cardiovascular disease, pneumonia and stroke may include increased length of hospital stay and a worse prognosis [35-37]. Thus, improvement of oral health education and services for the hospitalized elderly might decrease the incidence of periodontitis and it might concomitantly improve morbidity, mortality, and quality of life.

Acknowledgements This study was supported in part by the Japan Society for the Promotion of Science (JSPS) KAKENHI Grants (17K12067 to YO, 15K18973 to K.Suita, 17K11977 to MN, 17K17342 
to DU and 18K06862, 16H05300 to SO); the MEXT-Supported Program for the Strategic Research Foundation at Private Universities (S1511018 to SO); an Academic Contribution from Pfizer Japan (AC160910, AC1500818, AC170780 to SO); Mitsui Life Social Welfare Foundation (SO), and Research Promotion Grants from the Society for Tsurumi University School of Dental Medicine (29002, 27010 to IA, 28006 to NK, 28006 to YY, 29007 to K. Suita).

\section{Compliance with ethical standards}

Conflict of interest The authors report no conflict of interests.

\section{References}

1. Kinane DF, Stathopoulou PG, Papapanou PN (2017) Periodontal diseases. Nat Rev Dis Primers 3:17038

2. Eke PI, Dye BA, Wei L, Thornton-Evans GO, Genco RJ (2012) Prevalence of periodontitis in adults in the United States: 2009 and 2010. J Dent Res 91:914-920

3. Larsson L, Degens H, Li M, Salviati L, Lee YI, Thompson W, Kirkland JL, Sandri M (2019) Sarcopenia: aging-related loss of muscle mass and function. Physiol Rev 99:427-511

4. Frost RA, Nystrom GJ, Lang CH (2002) Lipopolysaccharide regulates proinflammatory cytokine expression in mouse myoblasts and skeletal muscle. Am J Physiol Regul Integr Comp Physiol 283:R698-R709

5. Bruunsgaard H, Andersen-Ranberg K, Jeune B, Pedersen AN, Skinhoj P, Pederson BK (1999) A high plasma concentration of $\mathrm{TNF}-\alpha$ is associated with dementia in centenarians. J Gerontol A Biol Sci Med Sci 54:M357-M364

6. Maggio M, Guralnik JM, Longo DL, Ferrucci L (2006) Interleukin-6 in aging and chronic disease: a magnificent pathway. $\mathrm{J}$ Gerontol A Biol Sci Med Sci 61:575-584

7. Graves DT, Cochran D (2003) The contribution of interleukin-1 and tumor necrosis factor to periodontal tissue destruction. J Periodontol 74:391-401

8. Sanchez P, Everett B, Salamonson Y, Ajwani S, George A (2017) Oral healthcare and cardiovascular disease: a scoping review of current strategies and implications for nurses. J Cardiovasc Nurs 32:E10-E20

9. Zhang J, Yu C, Zhang X, Chen H, Dong J, Lu W, Song Z, Zhou W (2018) Porphyromonas gingivalis lipopolysaccharide induces cognitive dysfunction, mediated by neuronal inflammation via activation of the TLR4 signaling pathway in C57BL/6 mice. J Neuroinflammation 15:37

10. Deleon-Pennell KY, de Castro Bras LE, Lindsey ML (2013) Circulating Porphyromonas gingivalis lipopolysaccharide resets cardiac homeostasis in mice through a matrix metalloproteinase9-dependent mechanism. Physiol Rep 1:e00079

11. Goodman CA, Frey JW, Mabrey DM, Jacobs BL, Lincoln HC, You JS, Hornberger TA (2011) The role of skeletal muscle mTOR in the regulation of mechanical load-induced growth. J Physiol 589:5485-5501

12. Bruusgaard JC, Egner IM, Larsen TK, Dupre-Aucouturier S, Desplanches D, Gundersen K (2012) No change in myonuclear number during muscle unloading and reloading. J Appl Physiol 113:290-296

13. Kiliaridis S, Engstrom C, Thilander B (1988) Histochemical analysis of masticatory muscle in the growing rat after prolonged alteration in the consistency of the diet. Arch Oral Biol 33:187-193

14. Okumura S, Takagi G, Kawabe J, Yang G, Lee MC, Hong C, Liu J, Vatner DE, Sadoshima J, Vatner SF, Ishikawa Y (2003)
Disruption of type 5 adenylyl cyclase gene preserves cardiac function against pressure overload. Proc Natl Acad Sci USA 100:9986-9990

15. Okumura S, Fujita T, Cai W, Jin M, Namekata I, Mototani Y, Jin H, Ohnuki Y, Tsuneoka Y, Kurotani R, Suita K, Kawakami Y, Hamaguchi S, Abe T, Kiyonari H, Tsunematsu T, Bai Y, Suzuki S, Hidaka Y, Umemura M, Ichikawa Y, Yokoyama U, Sato M, Ishikawa F, Izumi-Nakaseko H, Adachi-Akahane S, Tanaka H, Ishikawa Y (2014) Epacl-dependent phospholamban phosphorylation mediates the cardiac response to stresses. J Clin Invest 124:2785-2801

16. Yu H, He Y, Zhang X, Peng Z, Yang Y, Zhu R, Bai J, Tian Y, Li X, Chen W, Fang D, Wang R (2011) The rat IgGFc $\gamma B P$ and Muc2 C-terminal domains and TFF3 in two intestinal mucus layers bind together by covalent interaction. PLoS One 6:e20334

17. Cheema N, Herbst A, McKenzie D, Aiken JM (2015) Apoptosis and necrosis mediate skeletal muscle fiber loss in ageinduced mitochondrial enzymatic abnormalities. Aging Cell 14:1085-1093

18. Mahdy MAA (2018) Skeletal muscle fibrosis: an overview. Cell Tissue Res. https://doi.org/10.1007/s00441-018-2955-2

19. Cheng YC, Chen LM, Chang MH, Chen WK, Tsai FJ, Tsai CH, Lai TY, Kuo WW, Huang CY, Liu CJ (2009) Lipopolysaccharide upregulates uPA, MMP-2 and MMP-9 via ERK1/2 signaling in H9c2 cardiomyoblast cells. Mol Cell Biochem 325:15-23

20. Yoshimoto N, Togo S, Kubota T, Kamimukai N, Saito S, Nagao Y, Endo I, Sekido H, Nagashima Y, Shimada H (2005) Role of transforming growth factor- $\beta_{1}$ (TGF- $\left.\beta_{1}\right)$ in endotoxin-induced hepatic failure after extensive hepatectomy in rats. J Endotoxin Res 11:33-39

21. Khalil H, Kanisicak O, Prasad V, Correll RN, Fu X, Schips T, Vagnozzi RJ, Liu R, Huynh T, Lee SJ, Karch J, Molkentin JD (2017) Fibroblast-specific TGF- $\beta$-Smad2/3 signaling underlies cardiac fibrosis. J Clin Invest 127:3770-3783

22. Pavone LM, Rea S, Trapani F, De Pasquale V, Tafuri S, Papparella S, Oet Paciello (2012) Role of serotonergic system in the pathogenesis of fibrosis in canine idiopathic inflammatory myopathies. Neuromuscul Disord 22:549-557

23. Huang RL, Yuan Y, Zou GM, Liu G, Tu J, Li Q (2014) LPS-stimulated inflammatory environment inhibits BMP-2-induced osteoblastic differentiation through crosstalk between TLR4/MyD88/ NF-кB and BMP/Smad signaling. Stem Cells Dev 23:277-289

24. Dattaroy D, Seth RK, Sarkar S, Kimono D, Albadrani M, Chandrashekaran V, Hasson FA, Singh UP, Fan D, Nagarkatti M, Diehl AM, Chatterjee S (2018) Sparstolonin B (SsnB) attenuates liver fibrosis via a parallel conjugate pathway involving P53-P21 axis, TGF- $\beta$ signaling and focal adhesion that is TLR4 dependent. Eur J Pharmacol 841:33-48

25. Grumati P, Bonaldo P (2012) Autophagy in skeletal muscle homeostasis and in muscular dystrophies. Cells 1:325-345

26. Mialet-Perez J, Vindis C (2017) Autophagy in health and disease: focus on the cardiovascular system. Essays Biochem 61:721-732

27. Sato T, Ito Y, Nagasawa T (2015) Dietary L-lysine suppresses autophagic proteolysis and stimulates Akt/mTOR signaling in the skeletal muscle of rats fed a low-protein diet. J Agric Food Chem 63:8192-8198

28. Zhao WT, Yang M, Wu HM, Yang L, Zhang XM, Huang Y (2018) Systematic review and meta-analysis of the association between sarcopenia and dysphagia. J Nutr Health Aging 22:1003-1009

29. Kato S, Ekuni D, Kawakami S, Mude AH, Morita M, Minagi S (2018) Relationship between severity of periodontitis and masseter muscle activity during waking and sleeping hours. Arch Oral Biol 90:13-18

30. Ciciliot S, Rossi AC, Dyar KA, Blaauw B, Schiaffino S (2013) Muscle type and fiber type specificity in muscle wasting. Int $\mathbf{J}$ Biochem Cell Biol 45:2191-2199 
31. Jarvinen TA, Jarvinen TL, Kaariainen M, Kalimo H, Jarvinen M (2005) Muscle injuries: biology and treatment. Am J Sports Med 33:745-764

32. Kjaer M (2004) Role of extracellular matrix in adaptation of tendon and skeletal muscle to mechanical loading. Physiol Rev 84:649-698

33. Gillies AR, Lieber RL (2011) Structure and function of the skeletal muscle extracellular matrix. Muscle Nerve 44:318-331

34. Nichenko AS, Southern WM, Atuan M, Luan J, Peissig KB, Foltz SJ, Beedle AM, Warren GL, Call JA (2016) Mitochondrial maintenance via autophagy contributes to functional skeletal muscle regeneration and remodeling. Am J Physiol Cell Physiol 311:C190-C200

35. Yokota J, Ogawa Y, Yamanaka S, Takahashi Y, Fujita H, Yamaguchi N, Onoue N, Ishizuka T, Shinozaki T, Kohzuki M (2016)
Cognitive dysfunction and malnutrition are independent predictors of dysphagia in patients with acute exacerbation of congestive heart failure. PLoS One 11:e0167326

36. Kaysar M, Augustine T, Jim L, Benjamin C (2008) Predictors of length of stay between the young and aged in hospitalized community-acquired pneumonia patients. Geriatr Gerontol Int 8:227-233

37. Altman KW, Yu GP, Schaefer SD (2010) Consequence of dysphagia in the hospitalized patient: impact on prognosis and hospital resources. Arch Otolaryngol Head Neck Surg 136:784-789

Publisher's Note Springer Nature remains neutral with regard to jurisdictional claims in published maps and institutional affiliations. 